\title{
Social Media Chatbot for Increasing Physical Activity: Usability Study
}

\author{
Dillys LARBI ${ }^{\mathrm{a}, 1}$, Elia GABARRON ${ }^{\mathrm{a}, \mathrm{b}}$, Kerstin DENECKE $^{\mathrm{c}}$ \\ ${ }^{a}$ Norwegian Centre for E-health Research, Tromsø, Norway \\ ${ }^{\mathrm{b}}$ Faculty of Education, Østfold University College, Halden, Norway \\ ' Institute for Medical Informatics, Bern University of Applied Sciences, Bern, \\ Switzerland
}

\begin{abstract}
Fully automated self-help interventions integrated with social media chatbots could serve as highly cost-effective physical activity promotion tools for a large population. We have developed MYA, a Telegram-based chatbot for increasing physical activity. The objective of this study was to assess the usability of MYA. To identify usability issues, we recruited volunteers and asked them to interact with MYA and to answer the Chatbot Usability Questionnaire. Thirty volunteers participated in the study, $83.3 \%$ agreed MYA was welcoming during initial setup and $63.3 \%$ agreed MYA was very easy to use. MYA was perceived as realistic and engaging, easy to navigate, and its responses were useful, appropriate, and informative (all 53.3\%). However, $63.3 \%$ of respondents agreed MYA failed to recognize most of their inputs, and $43.3 \%$ claimed it would be easy to get confused when using MYA. Although the results are encouraging, it remains unclear if a social media chatbot can motivate people to increase their physical activity. MYA has the potential to do that, with improvements in functionalities like challenge personalization. The efficacy of these approaches should be studied in a clinical trial.
\end{abstract}

Keywords. Chatbot, social media, physical activity, health, participatory health

\section{Introduction}

The growing burden of chronic diseases highlights the urgent need to increase physical activity [1] through low-cost preventive interventions for large populations. Regular physical activity is important for disease prevention [1,2], better disease management, and improvement in overall health and quality of life [1]. However, engaging large adult populations in public health interventions for increasing exercise behavior remains challenging.

Digital technology interventions can successfully increase physical activity among adults [3-6]. Higher levels of engagement and effectiveness are associated with interventions that incorporate social media [7]. Fully automated self-help interventions integrated with social media chatbots could serve as highly cost-effective physical activity promotion tools. Chatbots are easy to use and do not require familiarity with a specific user interface. The use of a chatbot is mainly through a text-based dialogue where the communication aims to reach a goal, such as an increase in physical activity.

${ }^{1}$ Corresponding Author: Dillys Larbi, Norwegian Centre for E-health Research, University Hospital of North Norway, Sykehusveien. 239019 Tromsø, Norway; E-mail: dillys.larbi@ehealthresearch.no. 
Although the use of chatbots for health-related purposes is still an emerging field [8,9], its benefits related to increase in physical activity and user satisfaction have already been shown [10-12]. Thus, a chatbot on a social media platform (e.g., Telegram) could provide an inexpensive and widely available health intervention that appeals to a large population.

The objective of this study was to assess the usability of MYA, a social media-based chatbot prototype we designed to increase physical activity among adults.

\section{Methods}

\subsection{Development of the social media chatbot}

The chatbot was developed in 4 steps: strategy planning, design, implementation, and testing. As part of the strategy planning, we interviewed Psychology and Public Health experts about the intervention's requirements. The following functional requirements were specified: the chatbot should motivate a user to be physically active, communicate with a user, engage the user in an interesting conversation through a variety of conversation flows, have a rating feature, and offer exercise options. In addition, these non-functional requirements were specified: the chatbot should be rule-based, accessible through a social media platform, integrated with an activity tracker or step counter and retrieve the number of steps, and the chatbot/user dialogue should be stored and accessible. Furthermore, the chatbot should be designed as a friendly and empathic backslapper that informs a user of his/her current number of steps and encourages $\mathrm{him} /$ her to increase the daily step count.

We created a social media chatbot called MYA (Figure 1). To model the chatbot's input and its possible reactions, i.e., to create the dialogues, we used Business Process Model and Notation. Nine conversation flows were designed using Hillary Black's [13] conversation interface design template: first encounter, further encounter, menu, goals, challenges, steps today, facts, chatting, and help. The chatbot was developed using FlowXo [14], Google Sheets, and Telegram: a free cloud-based mobile and desktop social media app (Figure 2). FlowXo offers several functionalities to create, host, and maintain chatbots for social media platforms. The chatbot was integrated into Telegram using Botfather.

Flows, the "brain" of FlowXo chatbots, are activated by a specific trigger like a message sent by a user. This triggers MYA to send a message, picture, or video to the chat. The user can respond using either free text, predefined words, or button selection. The following information is gathered during the interaction and stored on Google Sheets with no encryption: username; current challenge and challenge number; current goal and goal number; daily step goal; and whether it is the first encounter. MYA currently has a simulated activity tracker, i.e., it has no integrated step counter. To make MYA more interesting, there is an integrated "small talk" functionality.

\subsection{Usability study}

We aimed to recruit at least 26 volunteers to test MYA, as suggested by Holmes et al. [15]. Volunteers aged 18 years or older were recruited from Bern University of Applied Sciences in Switzerland and Norwegian Centre for E-health Research and their affiliates.

Potential participants were contacted via e-mail or Telegram and invited to freely interact with MYA. They were asked to complete one or more of the following tasks: set 
a goal; request number of steps; a challenge; and to hear a fact. Participants then answered an anonymous online Microsoft Office Forms survey available in English, German, and Spanish. The survey included the Chatbot Usability Questionnaire (CUQ) [15], which includes questions about the chatbot's personality, onboarding, navigation, understanding, responses, error handling, and intelligence rated on a scale of 1 (strongly disagree) to 5 (strongly agree). We distinguished between the positive and negative items of the CUQ and analyzed the number and its corresponding percentage of respondents answering "Agree" and "Strongly agree" to these.

We summarized the survey and CUQ responses using descriptive statistics. Quantitative data were analyzed with SPSS (version 25; IBM Corp) whereas NVivo 12 Plus was used for the qualitative data (participant open feedback). All collected data were treated confidentially and only used for this study. The study protocol was assessed by the Cantonal Ethics Committee in Bern (BASEC-No: Req-2021-00244).

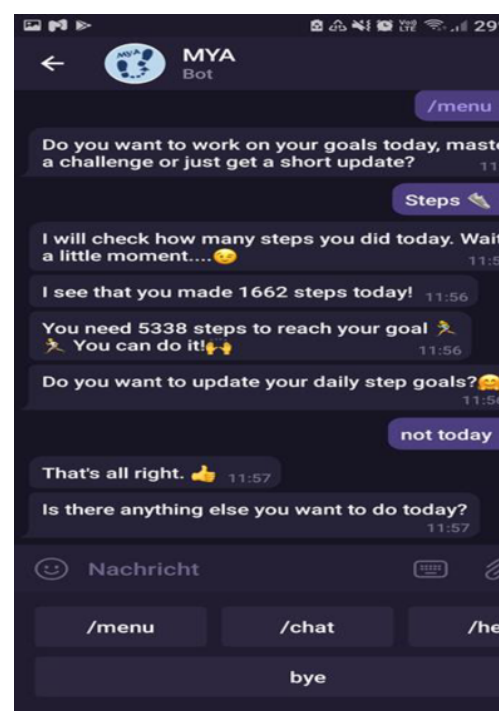

Figure 1. Screenshot of conversation between MYA (dark purple) and user (bright purple)

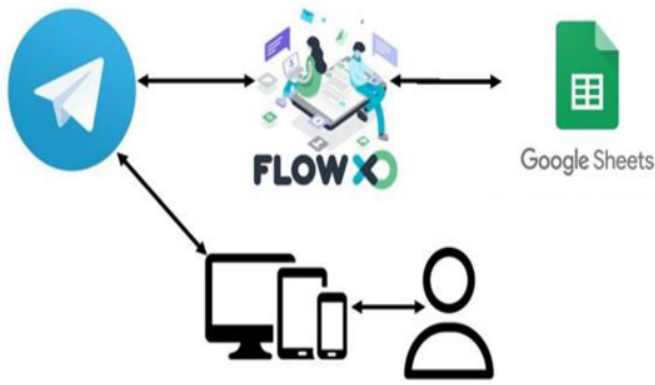

Figure 2. Technology stack of MYA

\section{Results}

Thirty volunteers, 15 males aged 18 to 69 years and 15 females aged 18 to 49 years, tested the chatbot and answered the survey. Nineteen participants $(19 / 30 ; 63.3 \%)$ reported a chatbot interaction time of $5-15$ minutes; 5 participants $(5 / 30 ; 16.7 \%)$ used it for $15-30$ minutes; 4 participants $(4 / 30 ; 13.3 \%)$ less than 5 minutes, and 2 participants $(2 / 30 ; 6.7 \%)$ used it for more than 60 minutes. To the question "Do you think MYA could help you in increasing your physical activity/change your activity behavior?", 16 of the respondents chose the option "maybe" (16/30; 53.3\%), while 7 answered "yes" and 7 answered "no" (both 7/30; 23.3\%).

The best quality attributed to MYA was that it was welcoming during the initial setup (83.3\% of respondents agreed or strongly agreed). The next best quality attributed to MYA was that it was very easy to use $(63.3 \%)$; followed by its personality was realistic 
and engaging, easy to navigate, and its responses were useful, appropriate, and informative (all 53.3\%). On the other hand, 63.3\% of the respondents thought MYA failed to recognize a lot of their inputs, and $43.3 \%$ thought it would be easy to get confused when using it (See Table 1).

Table 1. Participants who agreed or strongly agreed with the different items of the CUQ

\begin{tabular}{lc}
\hline "Positive" features of the chatbot & Agree/Strongly agree \\
\hline Q3-The chatbot was welcoming during initial setup & $25(83.3 \%)$ \\
Q15-The chatbot was very easy to use & $19(63.3 \%)$ \\
Q1-The chatbot's personality was realistic and engaging & $16(53.3 \%)$ \\
Q7-The chatbot was easy to navigate & $16(53.3 \%)$ \\
Q11-Chatbot responses were useful, appropriate, and informative & $16(53.3 \%)$ \\
Q9-The chatbot understood me well & $7(23.3 \%)$ \\
Q13-The chatbot coped well with any errors or mistakes & $6(20 \%)$ \\
Q5-The chatbot explained its scope and purpose well & $2(6.7 \%)$ \\
\hline "Negative" features of the chatbot & Agree/Strongly agree \\
\hline Q10-The chatbot failed to recognise a lot of my inputs & $19(63.3 \%)$ \\
Q8-It would be easy to get confused when using the chatbot & $13(43.3 \%)$ \\
Q2-The chatbot seemed too robotic & $11(36.7 \%)$ \\
Q14-The chatbot seemed unable to handle any errors & $9(30 \%)$ \\
Q12-Chatbot responses were not relevant & $7(23.3 \%)$ \\
Q6-The chatbot gave no indication as to its purpose & $6(20 \%)$ \\
Q16-The chatbot was very complex & $4(13.3 \%)$ \\
Q4-The chatbot seemed very unfriendly & $3(10 \%)$ \\
\hline
\end{tabular}

The last open-ended question allowed the respondents to write their general comments, including suggestions for improvement. In total, $73.3 \%(22 / 30)$ of the respondents wrote comments which can be grouped into 3 main themes: identified issues, preferred chatbot features, and suggestions for improvement. The identified issues with the chatbot, MYA, included: interaction difficulties; incomplete app design; spelling errors; and unresponsive/frozen app. The preferred features of MYA included the challenge feature and the goal feature. The participants suggested among others, a weekly challenge and wide subject variety for the 'small talk' feature.

\section{Discussion}

This study assessed the usability of MYA, a social media-based chatbot designed to increase physical activity using the Chatbot Usability Questionnaire (CUQ) [15]. More than $50 \%$ of the participants perceived MYA as realistic, engaging, and user-friendly. Compared with the negative features, the chatbot's positive features received higher ratings which suggest a positive participant attitude towards the social media chatbot. The positive attitudes could be a predictor of the chatbot's acceptability, as suggested by Nadarzynski et al. [16]. Like other chatbots aimed at improving physical activity [15, 22], MYA is integrated into a social media platform and does not need any installation, which eases access. MYA's ability to interact with users as a peer and remind them of their physical activity goals could contribute to continued chatbot use [17]. 
Further developed chatbot conversation flows, and its better interpretation of users' input might increase MYA's usability [15], which in turn could improve its acceptability and the users' confidence in its proper function. Fadhil et al. [18] associate a better chatbot experience that promotes user engagement with a well-designed and implemented chatbot. Since MYA was a prototype with a simulated activity tracker, the identification and reporting of issues by the participants was expected. These identified issues, together with the suggestions for improvement will help improve the features, functions, and design to provide a better user experience.

Approximately half of the participants responded that MYA could maybe help them to increase their physical activity. Among the remaining, half thought the chatbot could not help them while the other half thought it could. Most of the participants who responded that MYA could not help them, identified technical issues with the social media chatbot. Meanwhile those who responded that MYA could help increase their physical activity stated their preferred chatbot features and/or made suggestions for improvement. Previous studies on chatbots showed that they were effective in changing physical activity behavior [19-23]. However, there was a lack of sustained use and the chatbots failed to engage users [19]. Since an increased and sustained engagement has been reported among users of physical activity apps integrated with social media platforms [7], the integration of MYA with Telegram has the potential to engage users for continued use.

Limitations: Answers from the participants might not be comparable to the general population given the selection of participants, the majority of whom may be tech-savvy. Planned improvements include activity tracker integration and extension of MYA's rulebased communication knowledge to enable flexible conversations with users. Additional security issues (storage location, encryption, etc.) will be assessed and addressed. Further research on physical activity chatbots could explore the effect of additional functionalities, behavior change techniques, or its integration with different social media platforms.

\section{Conclusion}

Social media chatbots have the potential to increase physical activity among their users and through usability studies, all relevant features that increase user experience can be integrated. This paper presented usability test results of the initial prototype of MYA, a social media-based chatbot for increasing physical activity. Although the results are encouraging, it remains unclear if the chatbot can motivate people to increase their physical activity. MYA has the potential to do that with improvements in functionalities like challenge personalization. The efficacy of these approaches should be studied in future randomized controlled trials involving sedentary individuals.

\section{Acknowledgments}

The following Medical Informatics students at Bern University of Applied Sciences developed the chatbot as part of a student project: Patricia Romao, Stefanie Neuenschwander, and Apiwat-David Gaupp. We would also like to thank all the volunteers who participated in the study and Dr. Elisa Salvi for her feedback on the final draft. 


\section{References}

[1] World Health Organization. Global action plan on physical activity 2018-2030. More active people for a healthier world. 2018; Available from: https://apps.who.int/iris/bitstream/handle/10665/272722/9789241514187-eng.pdf.

[2] Ding D, Lawson KD, Kolbe-Alexander TL, et al. The economic burden of physical inactivity: a global analysis of major non-communicable diseases. Lancet. 2016;388(10051):1311-24.

[3] Kongstad MB, Valentiner LS, Ried-Larsen M, et al. Effectiveness of remote feedback on physical activity in persons with type 2 diabetes: A systematic review and meta-analysis of randomized controlled trials. J Telemed Telecare. 2019;25(1):26-34.

[4] Kramer LL, ter Stal S, Mulder BC, et al. Developing Embodied Conversational Agents for Coaching People in a Healthy Lifestyle: Scoping Review. J Med Internet Res. 2020;22(2):e14058.

[5] Laranjo L, Ding D, Heleno B, et al. Do smartphone applications and activity trackers increase physical activity in adults? Systematic review, meta-analysis and metaregression. Br J Sports Med. 2021;55(8):422-32.

[6] Tsoli S, Sutton S, Kassavou A. Interactive voice response interventions targeting behaviour change: a systematic literature review with meta-analysis and meta-regression. BMJ Open. 2018;8(2):e018974.

[7] Petersen JM, Prichard I, Kemps E. A Comparison of Physical Activity Mobile Apps With and Without Existing Web-Based Social Networking Platforms: Systematic Review. J Med Internet Res. 2019;21(8):e12687.

[8] Denecke K, Tschanz M, Dorner TL, et al. Intelligent Conversational Agents in Healthcare: Hype or Hope? Stud Health Technol Inform. 2019;259:77-84.

[9] Laranjo L, Dunn AG, Tong HL, et al. Conversational agents in healthcare: a systematic review. J Am Med Inform Assoc. 2018;25(9):1248-58.

[10] Bickmore TW, Silliman RA, Nelson K, et al. A randomized controlled trial of an automated exercise coach for older adults. J Am Geriatr Soc. 2013;61(10):1676-83.

[11] Gabarron E, Larbi D, Denecke K, et al. What Do We Know About the Use of Chatbots for Public Health? Stud Health Technol Inform. 2020;270:796-800.

[12] Kramer JN, Tinschert P, Scholz U, et al. A Cluster-Randomized Trial on Small Incentives to promote Physical Activity. Am J Prev Med. 2019;56(2):e45-e54.

[13] Black H. How to Design a Chatbot Script from Scratch. 2019; Available from: https://gumroad.com/1/conversationdesign

[14] Flow XO LLC. Create a chatbot with zero coding skills required. 2020; Available from: https://flowxo.com.

[15] Holmes S, Moorhead A, Bond R, et al. Usability testing of a healthcare chatbot: Can we use conventional methods to assess conversational user interfaces? Proceedings of the 31st European Conference on Cognitive Ergonomics; Association for Computing Machinery; 2019. p. 207-214.

[16] Nadarzynski T, Miles O, Cowie A, et al. Acceptability of artificial intelligence (AI)-led chatbot services in healthcare: A mixed-methods study. Digit Health. 2019;5:2055207619871808.

[17] Piao M, Kim J, Ryu H, et al. Development and Usability Evaluation of a Healthy Lifestyle Coaching Chatbot Using a Habit Formation Model. Healthc Inform Res. 2020;26(4):255-264.

[18] Fadhil A, Gabrielli S. Addressing Challenges in Promoting Healthy Lifestyles: The AI-Chatbot Approach. Pervasive Health. 2017.

[19] Zhang J, Oh YJ, Lange P, et al. Artificial Intelligence Chatbot Behavior Change Model for Designing Artificial Intelligence Chatbots to promote Physical Activity and a Healthy Diet: Viewpoint. J Med Internet Res. 2020;22(9):e22845.

[20] Kramer JN, Künzler F, Mishra V, et al. Which Components of a Smartphone Walking App Help Users to Reach Personalized Step Goals? Results From an Optimization Trial. Ann Behav Med. 2020;54(7):518-528.

[21] Kocielnik R, Xiao L, Avrahami D, et al. Reflection Companion: A Conversational System for Engaging Users in Reflection on Physical Activity. Proceedings of the ACM on Interactive, Mobile, Wearable and Ubiquitous Technologies. 2018;2:1-26.

[22] Piao M, Ryu H, Lee H, et al. Use of the Healthy Lifestyle Coaching Chatbot App to Promote StairClimbing Habits Among Office Workers: Exploratory Randomized Controlled Trial. JMIR Mhealth Uhealth. 2020;8(5):e15085.

[23] Maher CA, Davis CR, Curtis RG, et al. A Physical Activity and Diet Program Delivered by Artificially Intelligent Virtual Health Coach: Proof-of-Concept Study. JMIR Mhealth Uhealth. 2020;8(7):e17558. 